Proceedings

\title{
Equivalent Circuit-Based Open-Circuit Sensitivity Modelling of a Capacitive-Type MEMS Acoustic Sensor on Wafer Level ${ }^{\dagger}$
}

\author{
Jaewoo Lee *, Jong-Pil Im, Jeong-Hun Kim, Sol-Yee Lim and Seung-Eon Moon \\ ICT Materials \& Components Research Lab., ETRI, Daejeon 34129, Korea; jpim@etri.re.kr (J.-P.I.); \\ JeongHun@etri.re.kr (J.-H.K.); solyeeim@etri.re.kr (S.-Y.L.); semoon@etri.re.kr (S.-E.M.) \\ * Correspondence: jaewoo@etri.re.kr; Tel.: +82-42-860-5828 \\ † Presented at the Eurosensors 2018 Conference, Graz, Austria, 9-12 September 2018.
}

Published: 10 December 2018

\begin{abstract}
Equivalent circuit-based analytical open-circuit sensitivity modelling of a capacitive-type MEMS acoustic sensor for Internet of things (IoT) application is presented. It can not only evaluate simply the characteristic of the sensitivity on wafer level, but also improve the accuracy of the sensitivity due to including the fringing field between the diaphragm and each etching hole in the back-plate. The effective capacitor model is obtained by applying the approximately linearized electric-field method (ALEM), resulting in the equivalent circuit-based dynamic model. From the sensor with a $325 \mu \mathrm{m}$-radius diaphragm, the effective radius and the effective residual stress of the diaphragm were extracted to be $299 \mu \mathrm{m}$ and $+23.0 \mathrm{MPa}$, respectively. With the pull-in voltage of. $12.0 \mathrm{~V}$ and the pad capacitance of $0.23 \mathrm{pF}$; the open-circuit sensitivity was modelled to $11.3 \mathrm{mV} / \mathrm{Pa}$ at $1 \mathrm{kHz}$ in the bias of $10 \mathrm{~V}$.
\end{abstract}

Keywords: open-circuit sensitivity; equivalent circuit-based modelling; capacitive-type MEMS acoustic sensor; on wafer level

\section{Introduction}

For Internet of Things (IoT) application, Capacitive-type MEMS acoustic sensors have been investigated and developed in the field of various communication systems due to their matured competitiveness as the demand for integrated multifunction modules as well as their good frequency response [1-6]. Conventional MEMS acoustic sensors are usually packaged with read-out integrated circuits (ROICs) and subsequently, their sensitivities are generally evaluated on the basis of empirical data owing to the feature of a complicated capacitive-type transducer. However, despite the exact accuracy of the empirical data, their modelling or measurements are limited to some extent due to the intricate lumped parameters or the total package process. For this reason, on wafer level, equivalent circuit-based analytical open-circuit sensitivity modelling for capacitive-type MEMS acoustic sensor may be a promising characterization method for simple but proper investigation of a capacitive-type acoustic sensor itself.

In this paper, we report a simple equivalent circuit-based model of open-circuit sensitivity based on a couple of empirical data to accurately evaluate the open-circuit sensitivity of the acoustic sensor in a dynamic state on wafer level where lumped parameters of an equivalent circuit can be modelled using both the ALEM capacitor model [5] and the static characteristic of the pull in voltage. Thus, the equivalent circuit model conveniently offers open-circuit sensitivity for a capacitive-type acoustic sensor, resulting in the dynamic characterization on wafer level. 


\section{Equivalent Circuit-Based Model}

For evaluating dynamic response for a capacitive-type acoustic sensor, each lumped parameter of the equivalent circuit-based model is initially determined with few empirical data, where the model is largely composed of three parts: the capacitor model based on ALEM [5], the static opencircuit sensitivity model, and the dynamic open-circuit sensitivity model. Figure 1 shows schematic diagrams for the explanation of a unit cell of a capacitive-type sensor. In the process of the modelling, the sensor is split into each unit cell which can be divided into an inner part and an outer part. The width $\left(D_{a}\right)$ and the length $\left(D_{b}\right)$ of the outer part were $13 \mu \mathrm{m}$, respectively. Figure 2 describes the explanation of the ALEM-based capacitor model based on the vector notation. Through the ALEM capacitor model, a capacitor of an acoustic sensor with venting holes in the back plate can be separated into two capacitor: an intrinsic capacitor and a parasitic capacitor. From the sensor with a $325 \mu \mathrm{m}$-radius diaphragm, the effective radius and the effective residual stress of the diaphragm were extracted to be $299 \mu \mathrm{m}$ and $+23.0 \mathrm{MPa}$, respectively.

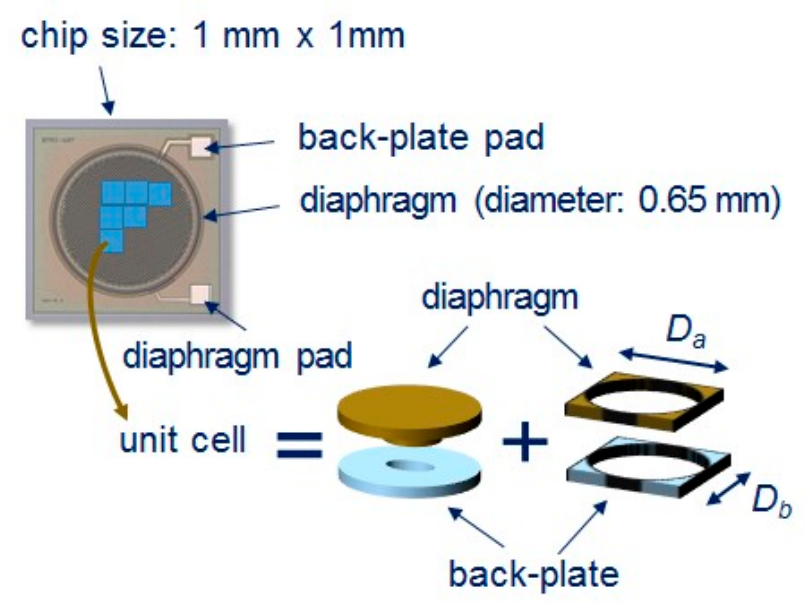

Figure 1. Schematic diagrams of a capacitive-type MEMS acoustic sensor for the explanation of a unit cell model which can be divided into an inner part and an outer part.

\section{ALEM-based Capacitor model}

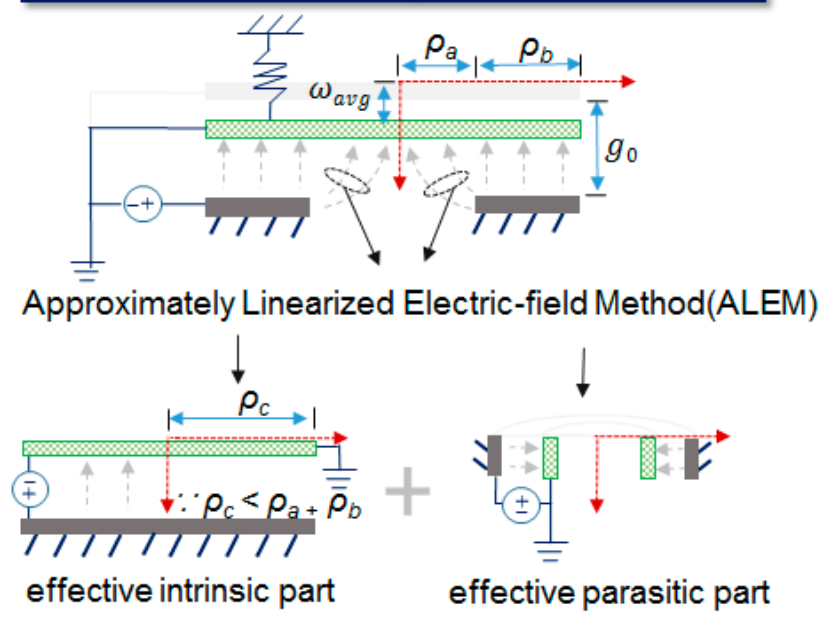

Figure 2. Schematic diagrams for the explanation of the ALEM-based capacitor model based on the vector notation.

\section{Results and Discussion}

As shown in Figure 3, measured impedance data were de-embedded to both the intrinsic and parasitic terms on the basis of the equivalent circuits for the acoustic sensor. Also, Figure 4 presents 
a structure-based analogous model for open-circuit sensitivity which is an equivalent circuit describing the interaction between the parameters in the three-signal domain [3,5,6]. Open-circuit sensitivity $\left(S_{o}\right)$ is defined to the ratio of the electrical output voltage $\left(v_{o u t}\right)$ to the input sound pressure as [5]

$$
S_{o}(\omega)=\frac{v_{\text {out }}(\omega)}{P(\omega)}=\frac{A_{\text {tran }}}{A_{\text {tran }}^{2} X(\omega)+Y(\omega)} \cdot \frac{\Gamma}{j \omega\left(C_{\text {int }}+C_{\text {par }}\right)}
$$

where $X(\omega)$ and $Y(\omega)$ are expressed by

$$
\begin{gathered}
X(\omega)=j \omega M_{r}+R_{r}(\omega)+R_{h}+R_{g}+\frac{1}{j \omega C_{b c}} \\
Y(\omega)=j \omega M_{d}+\frac{1}{j \omega C_{d}}+\frac{\Gamma^{2}}{j \omega\left(C_{i n t}+C_{p a r}\right)}
\end{gathered}
$$

Figure 5 shows modelled $S_{o}$ as a function of frequency and the resonance frequency $\left(f_{r}\right)$ at the given bias condition (air gap $=1.1 \mu \mathrm{m}$ ) for the capacitive-type MEMS acoustic sensor. With the pullin voltage of $12.0 \mathrm{~V}$ and the pad capacitance of $0.23 \mathrm{pF}$, the equivalent circuit-based open-circuit sensitivity in a dynamic response can be easily extracted, leading to $-38.9 \mathrm{dBV} / \mathrm{Pa}$ at $1 \mathrm{kHz}$ in the bias of $10 \mathrm{~V}$. Moreover, the resonance frequency $\left(f_{r}\right)$ was modelled to $74.2 \mathrm{kHz}$.

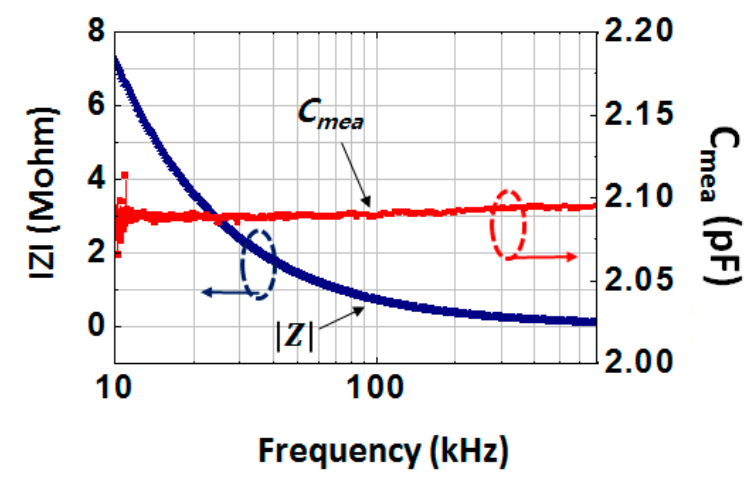

Figure 3. Measured capacitance and impedance data for the sensor used for extracting both the intrinsic and parasitic terms in the lumped equivalent circuit.

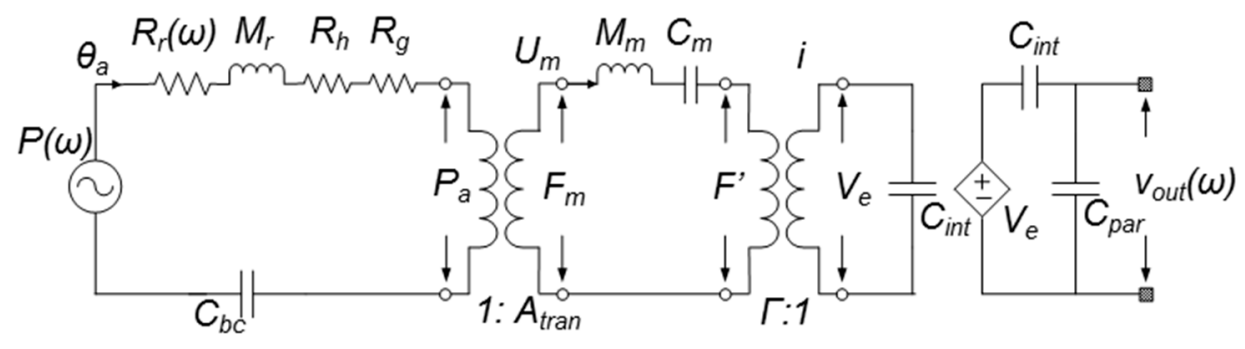

Figure 4. An equivalent circuit describing interaction between lumped parameters in the three-signal domain for a structure-based analogous model of open-circuit sensitivity. 


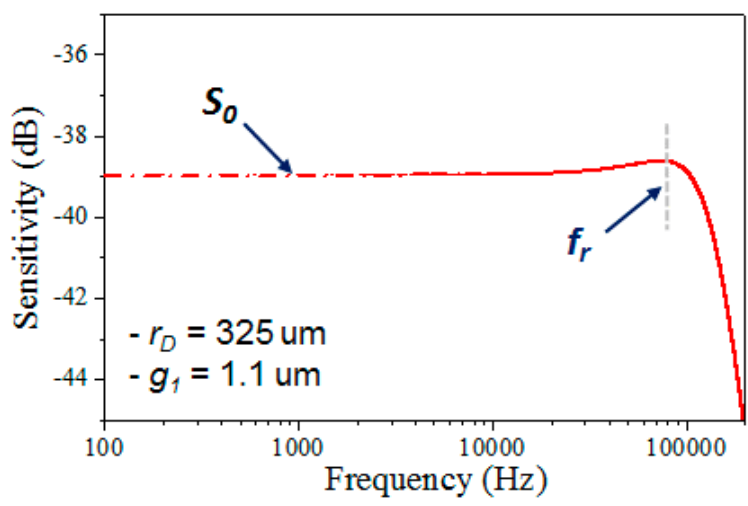

Figure 5. Modelled open-circuit sensitivity $\left(S_{0}\right)$ as a function of frequency and the resonance frequency $\left(f_{r}\right)$ at the given bias condition for the capacitive-type MEMS acoustic sensor.

\section{Conclusions}

An empirical-based dynamic model of open-circuit sensitivity for a capacitive-type MEMS acoustic sensor was proposed. With the capacitance model based on ALEM, the dynamic response of open-circuit sensitivity was characterized with the structure-based equivalent circuit as well as the analytic pull-in model. This lumped parameter-based model makes it possible to accurately evaluate the effective intrinsic capacitance, enabling its application for the frequency response of open-circuit sensitivity.

Author Contributions: J.-P.L. provided the main idea of modelling a capacitive-type MEMS acoustic sensor, designed and performed the experiments and wrote the paper. J.I. and J.-H.K. and S.-Y.L. and S.-E.M. contributed to the discussion of the obtained data and to the final version of the manuscript.

Acknowledgments: This work was supported by IITP grant funded by the Korea governments (2018 ICT R\&D Voucher, CMOS-MEMS Surface-type based ultra-small infrared detector integrated with ROIC for flame sensing of IoT).

\section{References}

1. Scheeper, P.R.; Donk, A.G.H.; Olthuis, W.; Bergveld, P. A Review of Silicon Microphones. Sens. Actuators A Phys. 1994, 44, 1-11.

2. Torkkeli, A.; Rusanen, O.; Saarilahti, J.; Seppa, H.; Sipola, H.; Hietanen, J. Capacitive microphone with lowstress polysilicon membrane and high-stress polysilicon backplate. Sens. Actuators A Phys. 2000, 85, 116-123.

3. Tilmans, H.A.C. Equivalent circuit representation of electromechanical transducers: I. Lumped-parameter systems. J. Micromech. Microeng. 1996, 9, 157-176.

4. Gato, M.; Iguchi, Y.; Ono, K.; Ando, A.; Takeshi, F.; Matsunaga, S.; Ya-suno, Y.; Tanioka, K.; Tajima, T. High-performance condenser microphone with single-crystalline silicon diaphragm and backplate. IEEE Sens. J. 2007, 7, 4-10.

5. Lee, J.; Jeon, J.H.; Je, C.H.; Kim, Y-G.; Lee, S.Q.; Yang, W.S.; Lee, J.S.; Lee, S.-G. A concave-patterned TiN/PECVD-Si3 $\mathrm{N}_{4} / \mathrm{TiN}$ diaphragm MEMS acoustic sensor based on a polyimide sacrificial layer. $J$. Micromech. Microeng. 2015, 25, 1-13.

6. Lee, J.; Jeon, J.H.; Kim, Y.-G.; Lee, S.Q.; Yang, W.S.; Lee, J.S.; Lee, S.-G. TiN/PECVD-Si3N/4/TiN diaphragmbased capacitive-type MEMS acoustic sensor. Electron. Lett. 2016, 52, 468-470.

(C) 2018 by the authors. Licensee MDPI, Basel, Switzerland. This article is an open access article distributed under the terms and conditions of the Creative Commons Attribution (CC BY) license (http://creativecommons.org/licenses/by/4.0/). 